\title{
Prüfenswerte Reformideen auf dem Boden der Realität
}

\section{Rudolf Luginbühl}

Rechtsanwalt

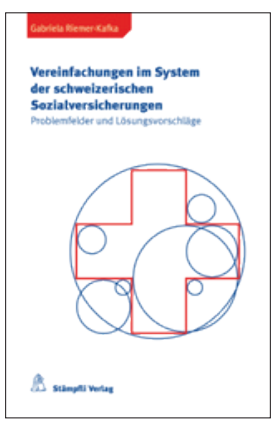

Gabriela

Riemer-Kafka

Vereinfachungen

im System der

schweizerischen

Sozialversiche-

rungen

Problemfelder und Lösungsvorschläge Bern: Stämpfli Verlag AG; 2014. 298 Seiten, 38 CHF. ISBN 978-3-7272-3121-6
Unser heutiges, kausales Sozialversicherungssystem funktioniert zwar grundsätzlich, doch bietet es viele Möglichkeiten zur Verbesserung. Es ist geprägt von verschiedenen Sozialversicherungszweigen, die teilweise gleichartige Leistungen erbringen. Das Koordinationsrecht hat einen hohen Stellenwert. Gerade der Arzt kennt die juristisch schwierige Unterscheidung zwischen Krankheit und Unfall und weiss um die Folgen, die es für einen Patienten haben kann, wenn die Versicherer nicht von einer Unfall-, sondern von einer Krankheitsbehandlung ausgehen.

Die Autorin, die als Ordinaria für Arbeits- und Sozialversicherungsrecht an der Universität Luzern tätig ist, schildert in verständlicher und präziser Sprache die Problemfelder des heutigen Systems und zeigt auf, wo Verbesserungsmöglichkeiten bestehen, deren Umsetzung machbar und kostenmässig tragbar ist. So schlägt sie keinen Neuaufbau des ganzen Systems im Sinne einer finalen Sozialversicherung vor, die beispielsweise das oben genannte Problem völlig lösen könnte. Sie bleibt auf dem Boden der Realität, was ihre Ideen und Vorstellungen umso prüfenswerter macht. Von anderen Reformvorschlägen unterscheiden sich ihre Ansätze insbesondere darin, dass sie sich nicht nur auf Teilaspekte, sondern auf die gesamte Sozialversicherung beziehen und zudem juristisch durchdacht und untermauert sind. Damit hebt sich das Werk wohltuend von blossen politischen Wunschvorstellungen irgendwelcher Couleur ab. Der gut gewählte Aufbau des Buches, der von den Grundlagen des heutigen Systems über dessen Problemfelder bis zu diskussionswerten Lösungsansätzen führt, ermöglicht die Lektüre in einem Zug. Nach einer kurzen Vorstellung der Reformthesen, deren Verbreitung das Buch dienen soll, folgen grundsätzliche Ausführungen, die zum besseren Verständnis des Zusammenspiels der Sozialversicherungen im heutigen System dienen. Diese Grundlagen sind knapp, klar und einleuchtend dargelegt. Der Sozialversicherungsexperte sieht so ihm bekannte Zusammenhänge in neuem Licht. Der Jurist freut sich über die vielen Referenzen und fachlichen Extras, die ihm seine Arbeit im Alltag erleichtern kön- nen und der Arzt lernt daraus, die Argumentation des Juristen nachzuvollziehen und erkennt die Hintergründe mancher Probleme, die er im Alltag erlebt. Das Buch erfüllt damit einen mehrfachen Zweck. Auf die Grundsätze des Systems folgt eine Darstellung der Problemfelder, d.h. der Bereiche, in denen unsere Sozialversicherung entweder nicht genügt, zu kompliziert ist oder sonst in der Praxis nicht überzeugt. In diesem Zusammenhang erwähnt die Autorin auch Uneinheitlichkeiten im Medizinalrecht. Der dritte Teil des Werks nimmt am meisten Raum ein. Hier geht es um die konkreten Reformvorschläge, die die Autorin in grosser Zahl vorlegt, ohne dabei das geltende System auf den Kopf zu stellen. Die Vorschläge sind ausformuliert und begründet. Bisher in Wissenschaft und Politik geäusserte Reformansätze werden erwähnt und gewürdigt. Wichtig sind der Autorin die Kostenfolgen, was die Anliegen umso prüfenswerter macht. Selbst Neuverteilungen im Bereich der sachlichen Zuständigkeiten, die sehr weit gehen können, sind diesem Ziel untergeordnet. Am Herzen liegen RiemerKafka nicht zuletzt Erleichterungen im Ablauf. Die Ärzteschaft dürfte sich angesprochen fühlen, sei es als Arbeitgeber oder als im Alltag unter administrativen Vorgaben leidende Fachleute. Riemer-Kafka spricht sich beispielsweise für eine Harmonisierung der Medizinaltarife unter den Sozialversicherungen aus.

\section{Die Autorin bleibt auf dem Boden der Realität, was ihre Ideen und Vorstellungen umso prüfenswerter macht.}

$\mathrm{Zu}$ Recht hat die Autorin ein grosses Informationsdefizit der versicherten Personen festgestellt. Auch dagegen finden sich Massnahmen. Kompetenzzentren sollen als regionale Anlaufstellen hilfesuchenden Bürgern den Zugang zur richtigen Sozialversicherung erleichtern. Es ist zu hoffen, dass der ganzheitliche Ansatz zu Reformen der Autorin und ihres Teams dazu führen wird, unser Sozialversicherungssystem noch effektiver, effizienter und transparenter zu gestalten. Die Gedanken wären es wert. 